\title{
LUTTINGER LIQUID AS A NEW METALLIC STATE OF CORRELATED FERMIONS
}

\author{
K. Byczuk
}

Institute of Theoretical Physics, Warsaw University, Hoża 69, 00-681 Warszawa, Poland

$$
\text { AND J. SPALEK }
$$

Institute of Physics, Jagiellonian University, Reymonta 4, 30-059 Kraków, Poland

This article provides an elementary introduction to the physics of the Luttinger liquid and summarizes its basic properties, as well as discusses examples, where such quantum liquid would be or has been observed experimentally. A comparison with the Fermi-liquid state is made

PACS numbers: 05.30.Fk

\section{Introduction}

In many three-dimensional fermionic systems their normal-phase properties are rather well described by the Landau-Fermi liquid theory. Numerous examples of such systems include: liquid ${ }^{3} \mathrm{He}$, simple metals, nuclear matter, neutron stars [1]. With some modifications, this theory encompasses also correlated metallic systems such as fermions close to the Mott localization or heavy fermions. On the other hand, recent progress in technology allowed us to synthesize systems which are effectively quasi two- or quasi-one-dimensional. For example, semiconductor heterostructures exhibiting fractional quantum Hall effect (FQHE) and high-temperature superconductors are two-dimensional metallic systems, whereas organic superconductors, inorganic salts, quantum wires, doped polyacetylene, edge excitations in FQHE systems are effectively one-dimensional structures dynamically [2]. Hence, most of the physical properties of the systems in lower dimensions are very unusual and almost impossible to understand within the Landau-Fermi liquid picture.

From the theoretical point of view, it is well established [2] that in one-dimensional systems of interacting fermions the Fermi liquid theory breaks down and the low-temperature properties of such metals are described by the Luttinger liquid. Probably, the edge excitations in FQHE heterostructures are the best known experimental realization of one-dimensional Luttinger liquid [3]. However, there is still an open question whether the interacting fermions in two dimensions can 
be described by the Fermi liquid theory or else, new ideas must be developed involving a multidimensional Luttinger liquid theory. Unusual physical properties of high-temperature superconductors in the normal state support the latter option [4].

In this contribution we present an elementary introduction to the Luttinger liquid theory in one and higher space dimensions. Of course, it will not be an exhaustive survey of this theory. Our purpose is to characterize the simplest and the most important similarities and differences between the Fermi and the Luttinger liquids. More comprehensive studies of these problems might be found by the reader in the recent review article [2], and the references cited therein.

\section{Landau-Fermi liquid theory}

We start with a discussion of the Fermi liquid theory along the line introduced by Landau [5]. He made two principal assumptions: (i) the interacting fermions in the ground state have the same symmetry as the ideal Fermi gas; and (ii) the low energy excitations of the interacting system can be labeled with the same quantum numbers as the energy levels of free gas. Physically, the first assumption means that we are dealing with interacting fermions in their normal state, with a well-defined Fermi surface at $T=0$. In other words, there is no phase transition in a system and the low-energy excitations are gapless (infinitesimally small amount of energy is sufficient to create them). The second supposition tells us that excitations of the system have charge $e$ and spin $\sigma$.

In order to introduce a concept of quasiparticle we must note that the low-energy spectrum does not depend on details of the interaction. Starting from an ideal gas of fermions we turn on the interaction so slowly that the system evolves adiabatically into a correlated state. And, eventually, this correlated state of physical fermions (electrons) can be equivalently described by a system of weakly interacting quasiparticles, which have the same quantum numbers as the initial particles. But one must bear in mind that the quasiparticles are not real electrons of the interacting system. They are interpreted as one-particle excitation quanta of the Fermi liquid and are introduced theoretically in order to describe the system of correlated electrons in a simple manner. Of course, real experiments are carried out on true electrons in the correlated state which, as we interpret sometimes, are dressed with a cloud of interaction and resemble quasiparticles (or vice versa, a quasiparticle looks like a dressed electron).

In the light of our above discussion, quasiparticle can be defined as follows: this is an exact low-energy elementary excitation of the Fermi liquid which has the properties of a real electron but its dynamical characteristics are modified and include all of the high-energy effects of the interaction.

The Landau assumptions imply that: (i) the entropy of the system is the same as that of a Fermi gas; (ii) the total number of quasiparticles is equal to the number of electrons (charge conservation); and (iii) the ground state of the Fermi liquid has the Fermi surface which includes the same volume in momentum space as that of the unperturbed system (Luttinger theorem). One can also show that the quasiparticle momentum distribution function has the Fermi-Dirac form, i.e. 


$$
n(p)=\frac{1}{\mathrm{e}^{\beta[\epsilon(p)-\mu]}+1},
$$

where $\beta=1 / k T, \mu$ is the chemical potential and $\epsilon(p, \sigma)$ is the dispersion relation of quasiparticles. Landau assumed the following form of it:

$$
\epsilon(p, \sigma)=\epsilon^{(0)}(p, \sigma)+\operatorname{Tr}_{\sigma^{\prime}} \int \mathrm{d} p^{\prime} f_{\sigma, \sigma^{\prime}}\left(p, p^{\prime}\right) \delta n\left(p, \sigma^{\prime}\right),
$$

where $\epsilon^{(0)}(p, \sigma)$ is the dispersion relation for free particles, $\delta n(p, \sigma)$ is a departure of the momentum distribution function of quasiparticles from that for noninteracting fermions and is supposed to be small. Finally, $f_{\sigma, \sigma^{\prime}}\left(p, p^{\prime}\right)$ is a Landau interaction function which describes all of the effects of scattering between the quasiparticles.

The Landau-Fermi liquid theory can be derived microscopically using one of the theoretical tools such as: the quantum field theory, the renormalization group, or the multidimensional bosonization. In fact, those methods are also useful in studying non-Fermi liquid systems. Their basic purpose is to determine the correlation functions, which provide information concerning the physical properties of the system. The most important correlation functions are: the one-particle (the propagator) and the two-particle (the four-point amplitude) Green functions. The propagator gives us all information on the single-particle operators, the particle distribution function, and the dispersion relation. On the other hand, the two-particle Green functions contain all information on the bosonic excitations in the system, the Landau interaction functions, as well as concerning other response functions needed in studying the transport properties of interacting particles.

In the following we briefly summarize the basic properties of the LandauFermi liquid theory. First, there is a sharp Fermi surface at $T=0$ where the electron momentum distribution function has a discontinuity. The jump of the distribution function is equal to $Z(0<Z \leq 1)$. The low-energy elementary excitations of the system are quasiparticles of charge $e$ and spin $1 / 2$. Their dispersion relation is visualized as the poles in the one-particle Green function, which has the universal form close to the Fermi surface, namely

$$
G^{\mathrm{FL}}(p, \omega)=\frac{Z}{\omega-\mu-v_{\mathrm{F}}\left(p-p_{\mathrm{F}}\right)+\mathrm{i} \gamma \mathrm{sgn}\left(p-p_{\mathrm{F}}\right)}+G_{\text {incoh }}(p, \omega) .
$$

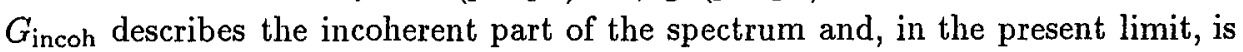
usually negligible. Here, $Z$ is interpreted as a renormalization of the electron wave function. The lifetime of quasiparticles is finite and is given by $\tau^{-1}=\gamma(\omega, T)=$ $a\left(\omega-\epsilon_{\mathrm{F}}\right)^{2}+b T^{2}$, where $a$ and $b$ are constants, and $\epsilon_{\mathrm{F}}$ is the Fermi energy. Note that at $T=0$ quasiparticles on the Fermi surface have an infinite decay time. In the time domain the one-particle propagator has the following form:

$$
G^{\mathrm{FL}}(p, t)=Z \mathrm{e}^{-\mathrm{i} \epsilon(p) t} \mathrm{e}^{-\gamma t}+G_{\text {incoh }}(p, t), \text { for }(t>0) .
$$

It oscillates with characteristic frequency $\epsilon(p)$ but also decays in time exponentially $\sim \mathrm{e}^{-\gamma t}$. For the excitations very close to the Fermi surface the damping is very small and then, the quasiparticles are well defined. Another important quantity is the spectral density function $A(p, \omega)=-\frac{1}{\pi} \operatorname{Im} G(p, \omega)$, which is equal to the probability that the one-particle excitation decays into eigenstates of the system. For an ideal gas $A(p, \omega) \sim \delta(\omega-\epsilon(p))$, whereas for the Fermi liquid it has a sharp peak (of finite width $\sim \gamma$ ) close to the Fermi energy. 
The next very important feature is that the Fermi liquid supports the bosonic excitations of zero energy (the zero sound, the spin waves, etc.) which can be visualized as the singularities of the four-point correlation functions. As a result, the physical properties of the Fermi liquid for $T \rightarrow 0$ are: (a) the linear specific heat is $C \sim \Gamma_{\mathrm{FL}} T$, where $\Gamma_{\mathrm{FL}}$ is proportional to the density of states at the Fermi energy; (b) the magnetic susceptibility is $\chi \sim$ const $+D T^{2}$; (c) the dc resistivity is $\rho \sim A T^{2}+\rho_{\text {res }}+\rho_{\mathrm{ph}}$, where $\rho_{\text {res }}$ is the residual part due to impurities, and $\rho_{\mathrm{ph}}$ is the phonon part; (d) the Hall coefficient $R_{\mathrm{H}}=1 /$ nec does not depend on the temperature.

We must emphasize that all the properties discussed so far are universal for all Fermi liquids and do not depend on details of the microscopic interaction.

\section{Haldane-Luttinger liquid theory}

Properties of interacting fermions in one spatial dimension are completely different from those in three dimensions. This is because the concept of the Fermi liquid is not applicable, since the electron-like quasiparticles do not exist [2].

In difference with higher dimensions, there are whole classes of one-dimensional models of correlated fermions, which can be solved exactly using the Bethe-ansatz wave function [2]. For example the Hubbard model, the Heisenberg model, the supersymmetric $t-J$ model, and their modifications including e.g. long-range hoppings or interactions. It is also possible to compute the long-time and large-distance correlation functions using asymptotically exact methods such as the bosonization transformation or the conformal field theory. By an asymptotically exact solution we mean the solution, which is rigorous at low energy (at low temperature) and at large distances. In that case only the long-wavelength fluctuations affect the system physical properties. Actually, the Landau-Fermi liquid is such an effective (asymptotically exact) theory.

In order to study correlated electrons in one dimension we start from the Tomonaga-Luttinger model. Namely, Tomonaga considered electrons moving along one-dimensional chain and assumed that their dispersion relation can be linearized close to the Fermi level. He introduced a cut-off (a band width) to limit the energy interval in which this approximation is valid. Later on, Luttinger started with a system of electrons with the linear dispersion relation, ranging from minus to plus infinities. The Hilbert space of such system is unbounded from below and there must be an infinite number of electrons piled up to the Fermi level. However, the spurious states do not affect the system low-energy properties. But, on the other hand, this kind of dispersion relation allowed him to formulate the noninteracting fermionic Hamiltonian (for particles at each branch $+k_{\mathrm{F}}$ and $-k_{\mathrm{F}}$ separately)

$$
H_{0}=\sum_{k \sigma} v_{\mathrm{F}}\left(k-k_{\mathrm{F}}\right) a_{k \sigma}^{+} a_{k \sigma}+\sum_{k \sigma} v_{\mathrm{F}}\left(-k-k_{\mathrm{F}}\right) b_{k \sigma}^{+} b_{k \sigma},
$$

in terms of bosonic density fluctuation operators

$$
H_{0}=\frac{\pi v_{\mathrm{F}}}{L} \sum_{k \sigma}\left[\rho_{a \sigma}(k) \rho_{a \sigma}(-k)+\rho_{b \sigma}(k) \rho_{b \sigma}(-k)\right] .
$$

It can be shown that the spectra of these two Hamiltonians are exactly the same. The advantage of this reformulation is that we.can add to the latter Hamiltonian 
an interacting part containing forward scatterings between electrons, which also have the form of the density-density fluctuations, i.e.

$$
\begin{aligned}
H_{\text {int }} & =g_{4} \sum_{k \sigma \sigma^{\prime}}\left[\rho_{a \sigma}(k) \rho_{a \sigma^{\prime}}(-k)+\rho_{b \sigma}(k) \rho_{b \sigma^{\prime}}(-k)\right] \\
& +g_{2} \sum_{k \sigma \sigma^{\prime}}\left[\rho_{a \sigma}(k) \rho_{b \sigma^{\prime}}(-k)+\rho_{b \sigma}(k) \rho_{a \sigma^{\prime}}(-k)\right] .
\end{aligned}
$$

The total Hamiltonian $H=H_{0}+H_{\text {int }}$ is bilinear in terms of density fluctuations operators and, therefore, it can be diagonalized exactly.

The properties of the Tomonaga-Luttinger model (and other models related to it) were investigated by many authors [2]. The most general feature is that the one-electron Green function has the form

$$
G(x, t) \sim \frac{1}{\sqrt{x-v_{\mathrm{F}}^{\mathrm{c} t}} \sqrt{x-v_{\mathrm{F}}^{\mathrm{s}} t}} \frac{1}{\left|x^{2}-\left(v_{\mathrm{F}}^{\mathrm{s}} t\right)^{2}\right|^{\alpha}},
$$

which proves that: (i) there is no electron-like quasiparticle (no single poles but only the branch cuts with an anomalous exponent $\alpha$ ); (ii) the spin and the charge degrees of freedom are separated (i.e. we encounter two Fermi velocities with $\left.v_{\mathrm{F}}^{\mathrm{s}} \neq v_{\mathrm{F}}^{\mathrm{c}}\right)$. Also, those studies showed that in one-dimensional systems an arbitrarily weak interaction destroys the Landau-Fermi liquid picture. In other words, such correlated systems cannot be obtained through an adiabatic evolution from the corresponding noninteracting gases.

We will discuss next a possibility of having a non-Fermi liquid in dimensions greater than one. In order to have such behavior in the normal phase it is necessary to have a singularity in the effective (Landau) interaction in the forward direction [6]. The physical origin of this singularity is widely discussed in the current literature. In order to investigate the basic properties of a non-Fermi liquid in two or three spatial dimensions we chose another route in our work [7]. Namely, we assumed at the beginning some form of the singular effective scattering amplitude between electrons of opposite spins and solved such model with the aid of the multidimensional bosonization technique. More precisely, our Hamiltonian has the following form:

$$
\begin{aligned}
H= & \sum_{S, \sigma} v_{\mathrm{F}}(S) \int \mathrm{d} \boldsymbol{x} \Psi_{\sigma}^{+}(S, x)\left(\frac{\widehat{n}_{S} \nabla}{\mathrm{i}}\right) \Psi_{\sigma}(S, x) \\
& +\sum_{S, T, \sigma, \sigma^{\prime}} \int \mathrm{d} \boldsymbol{x} f_{\sigma, \sigma^{\prime}}(S, T, x) \Psi_{\sigma}^{+}(S, x) \Psi_{\sigma}(S, x) \Psi_{\sigma^{\prime}}^{+}(T, x) \Psi_{\sigma^{\prime}}(T, x),
\end{aligned}
$$

where $T$ and $S$ label the separate points of the Fermi surface, $\widehat{n}_{S}$ is the normal vector to the Fermi surface at point $S, v_{\mathrm{F}}$ is the Fermi velocity, and $f_{\sigma \sigma^{\prime}}(S, T, x)$ is the scattering amplitude. The $f_{\uparrow \downarrow}(S, S, x)$ part of the interaction is singular in the thermodynamic limit, and the remaining regular parts were neglected for simplicity. Such assumption leads to the spin-charge separated Luttinger liquid in arbitrary spatial dimensions. For example, we find that the Green function has the form

$$
G(S, x, t) \sim \frac{1}{\sqrt{\widehat{n}_{S} x-v_{\mathrm{F}}^{\mathrm{c}} t} \sqrt{\widehat{\hat{n}}_{S} x-v_{\mathrm{F}}^{\mathrm{s} t}}}
$$


where $v_{\mathrm{F}}^{c, s}=v_{\mathrm{F}} \pm g$ are two different velocities and indicate the spin-charge decoupling. Of course, there are no quasiparticles with spin $\sigma$ and charge $e$. However, as we noted [7], it is possible to describe this system in terms of new pseudoparticles, i.e. holons and spinons, which have fermion-like properties (e.g. the commutation relations). They are the quanta of elementary excitations of this strongly correlated system and carry the charge $e$ and the spin $\sigma$ separately. As a result, the starting Hamiltonian (2) can be rewritten in the new (spinon-holon) representation in the following noninteracting Hamiltonian:

$$
H=\sum_{S, \boldsymbol{q}}\left[\boldsymbol{v}_{\mathrm{F}}^{\mathrm{c}}(S) q \Psi_{\mathrm{c}}^{+}(S, q) \Psi_{\mathrm{c}}(S, q)+v_{\mathrm{F}}^{\mathrm{s}}(S) q \Psi_{\mathrm{s}}^{+}(S, q) \Psi_{\mathrm{s}}(S, q)\right]
$$

The two fermionic Hamiltonians (2) and (3) have the same energy spectrum. However, the Hamiltonian (3) is very simple because it describes two ideal gases of holons and spinons. Additionally, we considered this model with an external magnetic field $h$, which produces the spin splitting of the carrier states. In any model with the spin-charge separation the presence of the magnetic field makes the situation more involved because it introduces a nontrivial interaction between the spinons and the holons. However, as we observed, such system can still be solved exactly. As a result we found the one-electron Green function

$$
G_{\sigma}(S, x, t) \sim \frac{1}{\left(\widehat{n}_{S} x-\lambda_{1} t\right)^{c_{\sigma}^{2} / 2}\left(\widehat{n}_{S} x-\lambda_{2} t\right)^{s_{\sigma}^{2} / 2}},
$$

where

$$
\lambda_{1,2}=(1 / 2)\left[v^{\mathrm{c}}+v^{\mathbf{s}} \pm \sqrt{\left(v^{\mathrm{c}}-v^{\mathrm{s}}\right)^{2}+4 h^{2}}\right]
$$

are the new characteristic Fermi velocities in this system (note their dependence on $h$ ). Also, the anomalous exponents

$$
c_{\sigma}^{2}=1-2 \sigma h / \sqrt{\left(v^{\mathrm{c}}-v^{\mathrm{s}}\right)^{2}+4 h^{2}}
$$

and

$$
s_{\sigma}^{2}=1+2 \sigma h / \sqrt{\left(v^{c}-v^{s}\right)^{2}+4 h^{2}}
$$

are functions of $h$. What is very surprising, we identify again the two new pseudoparticles except now, they have a fractional charge and a fractional spin.

The two models discussed briefly above provide examples of exactly solvable models, for which the correlated electrons do not resemble the Fermi liquid, although they still possess the Fermi surface obeying the Luttinger theorem. Following the suggestion due to Flaldane, such fermionic systems are called the Luttinger liquid. In the next part we briefly characterize it.

The general definition of the Luttinger liquid can be of the following form [8]: it is an effective state of interacting fermions possessing Fermi surface (at $T=0$ ) with volume obeying the Luttinger theorem, and for which there exists either an anomalous scaling of the spectral density function close to the Fermi surface (i.e. $A\left(\Lambda^{y_{1}}\left(k-k_{\mathrm{F}}\right), \Lambda^{y_{2}} \omega\right)=\Lambda^{y_{\mathrm{A}}} A\left(k-k_{\mathrm{F}}, \omega\right)$, where $y_{i}$ are arbitrary real numbers $)$, or the spin-charge separation (i.e. $v_{\mathrm{F}}^{\mathrm{c}} \neq v_{\mathrm{F}}^{\mathrm{s}}$ ). Note that the Landau-Fermi liquid represents special limit of the Luttinger liquid, i.e. if we have $y_{1}=y_{2}=-y_{\mathrm{A}}=1$, and $v_{\mathrm{F}}^{\mathrm{c}}=v_{\mathrm{F}}^{\mathrm{s}}$. 
The very important properties of the Luttinger liquid is the absence of a discontinuity in the momentum distribution function, i.e. very close to the Fermi surface we have that

$$
N\left(k-k_{\mathrm{F}}\right)=\frac{1}{2}-C \operatorname{sgn}\left(k-k_{\mathrm{F}}\right)\left|k-k_{\mathrm{F}}\right|^{\left(y_{2}+y_{A}\right) / y_{1}} .
$$

In other words, the renormalization factor $Z$ of an electron wave function vanishes.

In the Luttinger liquid of dimension $d>1$ the representative form of a one-electron Grien function may be taken as [9]:

$$
G_{\mathbf{R}}^{\mathrm{LL}}(k, \omega)=\frac{g(\alpha) \mathrm{e}^{-\mathrm{i} \pi \alpha}}{\omega_{\mathrm{c}}^{2 \alpha}\left(\omega-\epsilon_{k}^{\mathrm{c}}+\mathrm{i} \delta\right)^{1 / 2-\alpha}\left(\omega-\epsilon_{k}^{s}+\mathrm{i} \delta\right)^{1 / 2-\alpha}} ;
$$

where $\omega_{\mathrm{c}}$ is a cut-off (an energy scale within which this ansatz is valid), $g(\alpha)$ is a normalization constant, $\epsilon^{\mathrm{c}}$ and $\epsilon^{\mathrm{s}}$ are respectively the holon and the spinon dispersion relations, and $\alpha$ is the anomalous nonuniversal (depending on interaction) exponent. Here, there are no single poles (in distinction with the Fermi liquid Green function (1)), which means that there are no electron-like quasiparticles. Also, the time behavior of (4) is very different from that of (1), namely

$$
G_{\mathbf{R}}^{\mathrm{LL}}(k, t) \sim \frac{1}{t^{2 \alpha}} \mathrm{e}^{-\mathrm{i} \epsilon_{k} t},
$$

i.e. it decays according to the nonuniversal power law even for $k=k_{\mathrm{F}}$ ! Likewise, the spectral density function has a nonuniversal.power-law singularity at the Fermi surface $A\left(k_{\mathrm{F}}, \omega\right) \sim|\omega|^{2 \alpha-1}$, whereas far away from the Fermi surface it splits up into two singularities with a wide incoherent background in between. As a result, the density of states of the Luttinger liquid at the Fermi level is zero, i.e. $\rho(\omega) \sim|\omega|^{2 \alpha}$ for $\omega \rightarrow 0$.

Physical properties of the Luttinger liquid are as follows: (a) linear specific heat, $C \sim \Gamma_{\mathrm{LL}} T$ where now $\Gamma_{\mathrm{LL}} \sim 1 / v^{\mathrm{c}}+1 / v^{\mathrm{s}}$, (b) magnetic susceptibility $\chi \sim$ const $+d T^{2}$, (c) resistivity $\rho \sim A T^{1 \pm \epsilon}+\rho_{\text {res }}+\rho_{\text {ph }}$, and depends on the details of the interaction strength via $\epsilon$.

\section{Concluding remarks}

The theory predicts that in a strongly correlated electron system the Fermi liquid picture may be invalid. In its place, a new quantum (Luttinger) liquid could emerge with very unusual physical properties (note the anomalous scaling and the spin-charge separation).

The possibility of the Luttinger liquid appearance in physical systems is very exciting and poses some important questions. The Luttinger liquid behavior was observed in tunneling experiments between edge excitations in the heterostructures with FQHE [3]. Also, one-dimensional organic metals have spectral properties in agreement with the theory of Luttinger liquid [2]. However, there is still a heated debate in the literature related to the question whether electrons in quasi-two-dimensional high-temperature superconductors form the Luttinger liquid or not. We think it is crucial to understand their novel physics.

This work was supported by the Committee for Scientific Research through the grant No. 2 P03B 01310. 


\section{References}

[1] Windsurfing the Fermi Sea, Eds. T.T.S. Kuo, J. Speth, North-Holland, Amsterdam 1987.

[2] J. Voit, Rep. Prog. Phys. 57, 977 (1995).

[3] F.P. Milliken, C.P. Umbach, R.A. Webb, Solid State Commun. 97, 309 (1996).

[4] P.W. Anderson, Phys. Scr. Vol. T 42, 11 (1992); Science 256, 1526 (1992).

[5] E.G.D. Pines, P. Nozieres, Quantum Liquids, Vol. 1, W.A. Benjamin, New York 1966.

[6] P.W. Anderson, Phys. Rev. Lett. 65, 3206 (1990).

[7] K. Byczuk, J. Spałek, Solid State Commun. 99, 99 (1996).

[8] L. Yin, S. Chakravarthy, Int. J. Mod. Phys. B 10, 805 (1996).

[9] K. Byczuk, J. Spałek, W. Wójcik, unpublished. 